\title{
A Study on Energy Consumption in WLAN and Improving its Efficiency Through an NBE-Algorithm
}

\author{
Anupam Das \\ Asst. Professor \\ Dept. of Computer Sc. \& IT \\ Cotton College, Assam, India
}

\author{
Shikhar Kr. Srama \\ Prof. and Head \\ Dept of Computer and Information Technology \\ Gauhati University, Assam, India
}

\begin{abstract}
The primary aim of this work is to enhance the energy conservation. This can be achieved by reducing the spending of energy in the network, i.e. in the considered WLAN. One algorithm is proposed in this study which is based on energy for the different needs of the network. In the proposed study, the movements of the wireless nodes yield in old routingpaths, due to the lack of route accumulation updating. For that, a new cross layer framework is implemented along with the proposed algorithm to improve route supply performance in AODV. Using simulation, the results found for the proposed algorithm and the results show that it achieves better performance than the existing methods.
\end{abstract}

\section{General Terms}

NBE-Algorithm which is used to enhance the Energy Conservation and thus making the network more efficient in terms of Energy Consumption

\section{Keywords}

NBE (Need Based Energy), PDR (Packet Delivery Ratio), E2E Delay (End to End Delay), WLAN (Wireless LAN),.

\section{INTRODUCTION}

\subsection{What is WLAN?}

The basic definition of WLAN is the collection of mobile nodes with a centralized infrastructure, i.e. Access-Pont (AP).

\subsection{Assumptions for the study:}

The fundamental assumption or supposition is that the nodes involved in the Network i.e. in the WLAN will always same type of packets and spend same amount of energy to forward any packet and also data rate will be fixed in the network. Generally, the nodes in any WLAN collect route information through overhearing and accumulate those in route caches by using AODV protocol. These nodes devour power unnecessarily due to overhearing the transmissions of their neighbors. For this reason, the network performance is enhanced but more energy consumption occurs unnecessarily. The study aims to reduce the effect of spending energy with the help of the proposed algorithm Need Based Energy (NBE) algorithm. The movements of the nodes yield in old routingpaths, due to the lack of route accumulation updating. For that, a new cross layer framework is implemented along with the NBE to improve route supply performance in AODV. Using simulation, the results found for the proposed algorithm shows that it achieves better performance than the existing methods.

\subsection{Proposed study includes the following in the WLAN model:}

a) Sending beacon message to check the nodes availability

b) Maintaining Routing table

c) Select the path based on the energy level from routing table

d) Choose the best quality of service parameter

e) Implemented message broad casting to minimize packet loss

f) Increase the quality of service

g) Increase the efficiency in energy consumption

h) Increase the delivery ratio and decrease the delay and packet loss

\section{ENERGY MODEL}

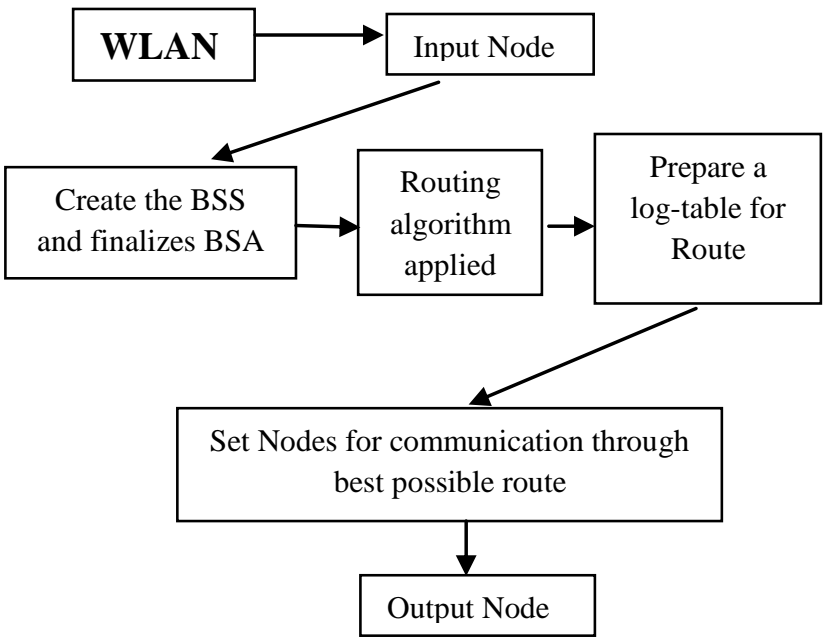

Figure-1: Energy Model

In the energy computation, a simple energy model is used where every node or station initializes with an initial energy and while transmitting any packet from one node to another it consumes one unit of energy. To organize the entire work four module are used. 


\subsection{BBCM (Basic-Service-Set, Basic-} Service-Area Creation Module):

This module creates the BSS and BSA. The module takes two parameters data rate and number of nodes.

\subsection{AOM (Address Organization Module):}

This module takes address of each node and specifies the source and destination for sending and receiving data. Message is sent to each node randomly.

\subsection{LPM (Log-Preparing-Module):}

This module takes location information of the network and prepares a log-table along with the necessary information its weights. Then it selects the next hop on the basis of its energy consumption. It does by calculating the average distance of all the neighbouring nodes and checks their energy level. Finally it selects all such nodes which are active and having maximum energy with less than or equal to the average distance among its entire neighbours.

\subsection{HDMGM (Hop Destination Map Generator Module):}

This module forwards the packets to other nodes and generates the next hop destination map which is the result of the routing algorithm we used and forwards the received packets to the selected nodes. This gives the entire information to each nodes about its own as well as all the possible neighbouring nodes along with their energy level. This information updates after each transmission. Finally the module gives the output nodes in the network.

\section{ANALYSIS}

The following QoS metrics are used to analyze the performance of the network of the Existing and Proposed Model:

End-to-End Delay (E2E Delay)

PDR (Packet Delivery Ratio)

Packet Lost

Energy Consumption

\subsection{E2E Delay}

The basic definition of End-to-end Delay in network is the average time taken by a data packet to arrive in the destination. It also includes the delay caused by route discovery process and the queue in data packet transmission. Only the data packets that successfully delivered to destinations that counted.

E2E Delay $=\sum($ arrive time - send time $) / \sum$ Number of connections

The interpretation of E2E delay is the lower value of end to end delay means the better performance of the protocol. Hence our proposed model shows better performance as the end-to-end delay differs with the proposed system in a quite distinctly as in the existing the existing system end-to-end delay is quite big in terms of the proposed system. During the time progresses the network shows quite a distinct packet delay. The following are some set of values given for immediate comparison and the graph generated by ns 2 shows how better the proposed system worked.
Table-1: Data of End to End delay

\begin{tabular}{|l|l|l|}
\hline Delay & \multicolumn{1}{|c|}{ Packet Size } & \multicolumn{1}{c|}{ Packet Size } \\
\hline 0.0 & 0.0 & 0.0 \\
\hline 0.5 & 0.02 & 0.01 \\
\hline 0.8 & 0.06 & 0.03 \\
\hline 0.85 & 0.09 & 0.04 \\
\hline 0.9 & 0.16 & 0.07 \\
\hline 1.0 & 0.25 & 0.08 \\
\hline
\end{tabular}

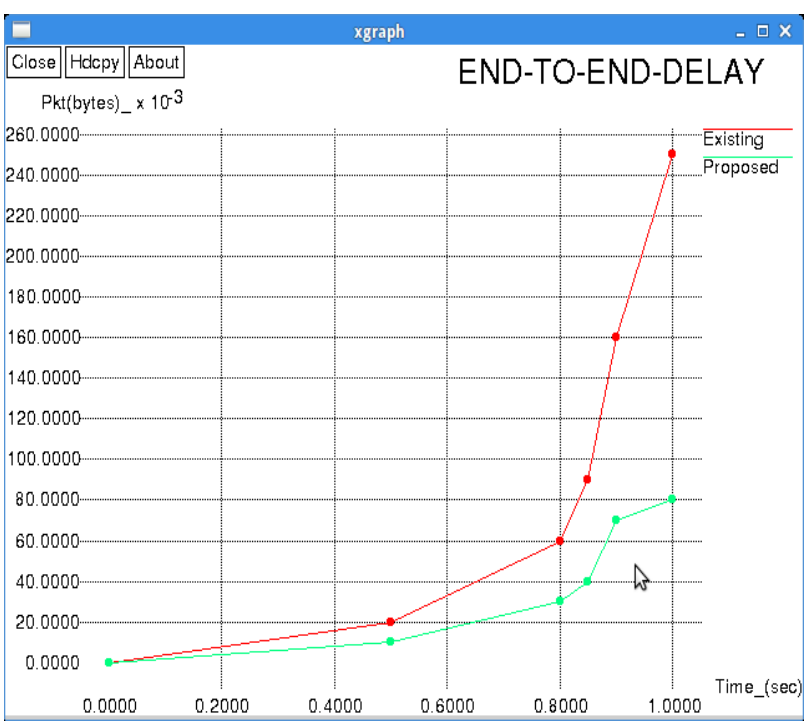

Figure-2: End-to-End delay

\subsection{PDR (Packet Delivery Ratio):}

The meaning of Packet delivery ratio in terms of network performance metric is the ratio of the number of delivered data packet to the destination. This actually illustrates the level of delivered data to the destination i.e.

\section{PDR $=$ Sum of all Number of packets received $/$ Sum of all} Number of packets sent

The interpretation of PDR is that the greater value of packet delivery ratio means the better performance of the protocol. Hence, the PDR shows in our proposed model is better as PDR differs with the proposed system in a quite distinctly as in the existing the existing system. The PDR is quite high in terms of the proposed system.

The following are some set of values given for immediate comparison and the graph generated by ns 2 shows how better the proposed system worked. 
Table-2: Data of PDR

\begin{tabular}{|l|l|l|}
\hline Delay & Existing System & \multicolumn{1}{c|}{ Proposed System } \\
\hline 0.0 & 0 & 0 \\
\hline 0.5 & 27 & 45 \\
\hline 0.8 & 58 & 84 \\
\hline 0.85 & 79 & 105 \\
\hline 0.9 & 96 & 135 \\
\hline 1.0 & 155 & 175 \\
\hline
\end{tabular}

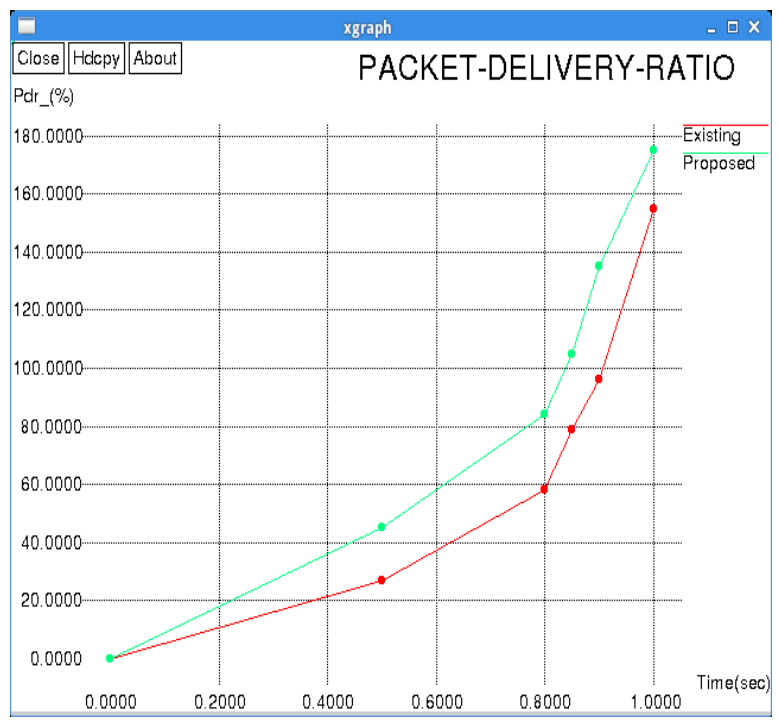

Figure-3: PDR Comparison

\subsection{Packet Lost}

The Packet Lost in any network means the total number of packets dropped during the simulation.

Packet lost $=$ Number of packet send - Number of packet received.

The analytical interpretation of packet lost is that the lower the value of the packet lost means the better performance of the protocol. The following are some set of values given for immediate comparison and the graph generated by ns 2 shows how better the proposed system worked

\section{Table-3: Data of Packet Lost}

\begin{tabular}{|l|l|l|}
\hline Delay & Existing System & \multicolumn{1}{c|}{ Proposed System } \\
\hline 0 & 0 & 0 \\
\hline 5 & 1 & 1 \\
\hline 10 & 1.16 & 1.01 \\
\hline 15 & 1.6 & 1.3 \\
\hline 20 & 1.9 & 1.5 \\
\hline 25 & 2.2 & 1.7 \\
\hline
\end{tabular}

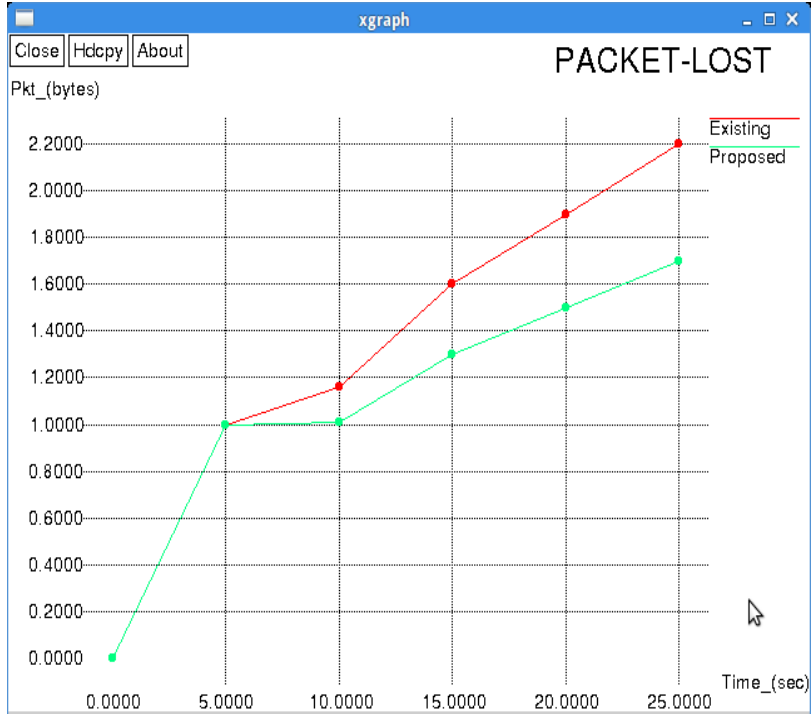

\section{Figure-4: Packet Lost comparison}

\subsection{Energy Consumption}

The energy consumption in a network means the amount energy spent by all the nodes involved in the network during the different activities like packet sending, packet receiving, or even the nodes consume energy during their different modes they are in the network in the entire session.

\section{Table-4: Data of Energy Conservation}

\begin{tabular}{|l|l|l|}
\hline Delay & Energy Conserved & Energy Conserved \\
\hline 0 & 1000 & 1000 \\
\hline 25 & 952 & 992 \\
\hline 50 & 895 & 923 \\
\hline 75 & 812 & 876 \\
\hline 100 & 715 & 815 \\
\hline 150 & 612 & 762 \\
\hline 200 & 512 & 727 \\
\hline
\end{tabular}

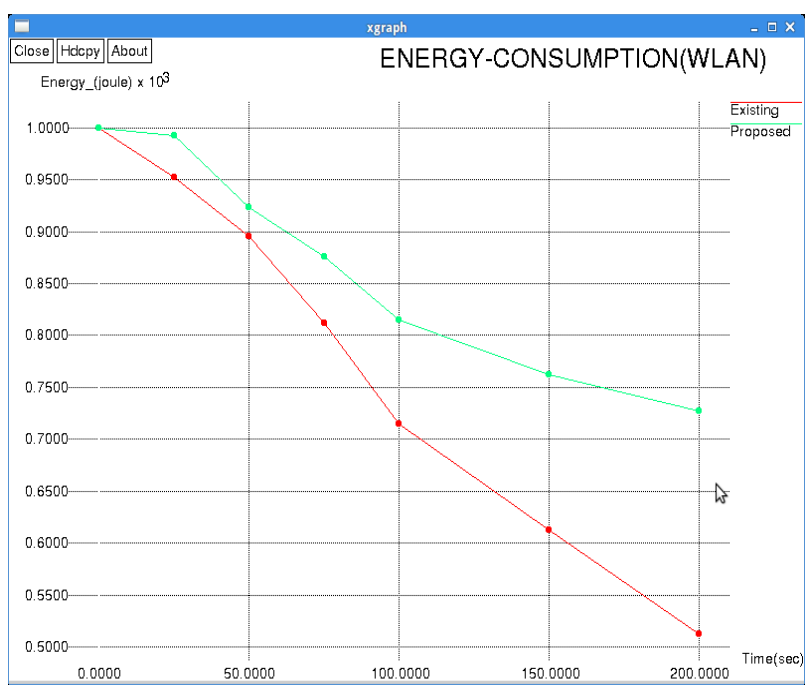

Figure-5: Energy Conservation comparison 


\section{CONCLUSION}

In this study, the performance of the WLAN is minutely scrutinized with the help of many parameters like End-to-End delay, PDR, Packet-Lost and then the consumption of energy in the network is computed. During our study, we used the existing protocol with access points and then our proposed algorithm NBE is implemented in the model and results are found distinctly better in all the parameters we considered as metrics of the performance of the network. In fig-2, the Endto-End delay is shown and clearly the proposed algorithm showed far better performance than the exiting one. In fig-3, the packet-lost is shown and clearly the proposed algorithm showed far better performance than the exiting one. In fig-4, the PDR is shown and clearly the proposed algorithm showed outstanding performance as compared to the exiting one. In fig-5, the Energy-Consumption is shown and the proposed algorithm showed a very consistent performance than the exiting one. Therefore, we can conclude that the algorithm proposed by us enhances the performance of the different parameters along with this it increases the efficiency in the energy consumption in the network.

\section{REFERENCES}

[1] Energy Efficiency of Ad Hoc Wireless Networks with Selfish Users By Vikram Srinivasan $\square$, Pavan Nuggehalli $\square$, Carla F. Chiasserini_, Ramesh R. Rao.

[2] Wireless LAN Performance Under Varied Stress Conditions in Vehicular Traffic Scenarios By Jatinder Pal Singh, Nicholas Bambos and Bhaskar Srinivasan and Detlef Clawin Robert Bosch Corporation
[3] A cross-cultural analysis of available evidence on the social uses of wireless communication technology By Manuel Castells (USC), Mireia Fernandez-Ardevol (UOC), Jock Linchuan Qiu (USC), and Araba Sey (USC)

[4] Energy Efficiency of the IEEE 802.15.4 Standard in DenseWirelessMicrosensorNetworks:Modeling and Improvement Perspectives By Bruno Bougard13, Francky Catthoor13 1IMEC, Leuven Belgium \{bougardb, catthoor\}@imec.be Denis C. Daly2, Anantha Chandrakasan2 2Department of EECS, Massachusetts Institute of Technology, Cambridge, MA 02139 \{ddaly, anantha\}@mit.edu Wim Dehaene3 3Department of EE, K.U. Leuven, Belgium wim.dehaene@esat.kuleuven.ac.be

[5] MODELING ENERGY EFFICIENT SECURE WIRELESS NETWORKS USING NETWORK SIMULATION Ramesh Karri and Piyush Mishra Department of Electrical and Computer Engineering Polytechnic University, Brooklyn, NY, 11201

[6] Energy Efficiency Analysis of IEEE 802.11 DCF with variable Packet Length was carried out by Bo $\mathrm{Gao}^{1}$, Yuhang ${ }^{1}$ Yang and Huiye $\mathrm{Ma}^{2}{ }^{1}$ Dept. of Electronic Engineering, Shanghai Jiao Tong University, Shanghai China and ${ }^{2}$ Dept. of Computer Science and Engineering, Chinese University of Hong Kong

[7] Jean Lorchat and Thomas Noel worked on Energy efficiency and QoS optimization of IEEE 802.11 communication using frame aggregation. 